\title{
Aerobic capacities in the skeletal muscles of Weddell seals: key to longer dive durations?
}

\author{
S. B. Kanatous ${ }^{1}$, R. W. Davis ${ }^{2}$, R. Watson ${ }^{2}$, L. Polasek ${ }^{2}$, T. M. Williams ${ }^{3}$ and O. Mathieu-Costello ${ }^{4}$ \\ ${ }^{1}$ Department of Internal Medicine, University of Texas Southwestern Medical Center, Dallas, TX 75390, USA, \\ ${ }^{2}$ Department of Marine Biology, Texas A\&M University, Galveston, TX 77553, USA, ${ }^{3}$ Department of Biology, \\ University of California Santa Cruz, Santa Cruz, CA 95604, USA and ${ }^{4}$ Department of Medicine, \\ University of California San Diego, San Diego, CA 92093, USA \\ *Author for correspondence (e-mail: shane.kanatous@utsouthwestern.edu)
}

Accepted 20 August 2002

\begin{abstract}
Summary
In contrast to terrestrial animals that function under hypoxic conditions but display the typical exercise response of increasing ventilation and cardiac output, marine mammals exercise under a different form of hypoxic stress. They function for the duration of a dive under progressive asphyxia, which is the combination of increasing hypoxia, hypercapnia and acidosis. Our previous studies on short-duration, shallow divers found marked adaptations in their skeletal muscles, which culminated in enhanced aerobic capacities that are similar to those of athletic terrestrial mammals. The purpose of

for morphometric analysis, enzymology, myoglobin concentrations and fiber-type distribution. The results showed that the skeletal muscles of Weddell seals do not have enhanced aerobic capacities compared with those of terrestrial mammals but are adapted to maintain low levels of an aerobic lipid-based metabolism, especially under the hypoxic conditions associated with diving. The lower aerobic capacity of Weddell seal muscle as compared with that of shorter-duration divers appears to reflect their energy-conserving modes of locomotion, which enable longer and deeper dives.
\end{abstract} the present study was to assess the aerobic capacity of skeletal muscles from long-duration divers. Swimming and non-swimming muscles were collected from adult Weddell seals, Leptonychotes weddelli, and processed
Key words: Weddell seals, Leptonychotes weddelli, aerobic capacity, diving, skeletal muscle, mitochondria.

\section{Introduction}

Marine mammals exhibit an extraordinary ability to prevent the deleterious effects of hypoxia, ischemia and reperfusion associated with breath-hold diving. In contrast to terrestrial animals that function under hypoxic conditions but display the typical exercise response of increasing ventilation and cardiac output, marine mammals exercise under a different form of hypoxic stress. They function for the duration of a dive under progressive asphyxia, which is the combination of increasing hypoxia, hypercapnia and acidosis (Elsner et al., 1998; Hochachka, 1986, 1992; Kanatous et al., 1999). Consequently, the amount of oxygen stored in their blood and muscles (i.e. total body oxygen stores) and the rate of oxygen use by tissues and organs are the primary factors controlling aerobic dive duration (Burns, 1999; Kooyman and Ponganis, 1998). Previous studies have shown that airbreathing, diving vertebrates have a number of physiological adaptations in their muscles (and other tissues) that allow them to sustain an aerobic, lipid-based metabolism under conditions of hypoxia and ischemia. Relative to terrestrial mammals, our previous studies of skeletal muscles of harbor seals (Phoca vitulina), Northern fur seals (Callorhinus ursinus) and Steller sea lions (Eumetopias jubatus) have shown that these muscle adaptations include: (1) an enhanced aerobic capacity resulting from increased volume density of mitochondria and citrate synthase activity, (2) a greater reliance on fatty acid catabolism for aerobic ATP production based on an increased $\beta$-hydroxyacyl CoA dehydrogenase activity, (3) enhanced oxygen storage and diffusion capacity resulting from an increased myoglobin concentration and volume density of mitochondria, and (4) a reduced dependency on blood-borne oxygen and metabolites resulting from a decreased capillary density (Davis et al., 1991; Davis and Kanatous, 1999; Guyton et al., 1995; Kanatous et al., 1999, 2001; Kooyman et al., 1999). These adaptations increase their aerobic dive limit (ADL), which is the longest dive that an animal can make while relying primarily on oxygen stored in the blood and muscle to sustain aerobic metabolism.

Despite the marked adaptations for hypoxia resistance demonstrated by harbor seals, Steller sea lions and Northern 
fur seals, these species are relatively short-duration divers (average dive durations are approximately $2.5 \mathrm{~min}$ ). In comparison, Weddell seals (Leptonychotes weddelli) represent an elite diving species that may remain submerged for over $60 \mathrm{~min}$. These seals are highly adapted for an aquatic life in the shore-fast and pack-ice habitats of Antarctica (Kooyman, 1981). Deep foraging dives are usually 100-350 m deep (maximum recorded depth of $741 \mathrm{~m}$ ) and between 15-20 min in duration (maximum recorded duration of $82 \mathrm{~min}$ ) (Davis et al., 1999; Castellini et al., 1992; Kooyman, 1981; Testa, 1994; Wartzok et al., 1992). The majority of free-ranging dives by Weddell seals is within their ADL and suggests an exceptional ability to tolerate prolonged periods of apnea.

The purpose of the present study was to assess the aerobic capacity of the skeletal muscles of deep, long-duration divers. We hypothesized that skeletal muscle of Weddell seals would exhibit the same, or an even greater, increase in their capacity for aerobic, lipid-based metabolism than we had observed in the comparatively shallow, short-duration divers. However, we found that the skeletal muscles of Weddell seals do not have enhanced aerobic capacities compared with those of terrestrial mammals. This may result from energyconserving modes of locomotion employed by Weddell seals that permit prolonged dive durations but at overall low levels of metabolism.

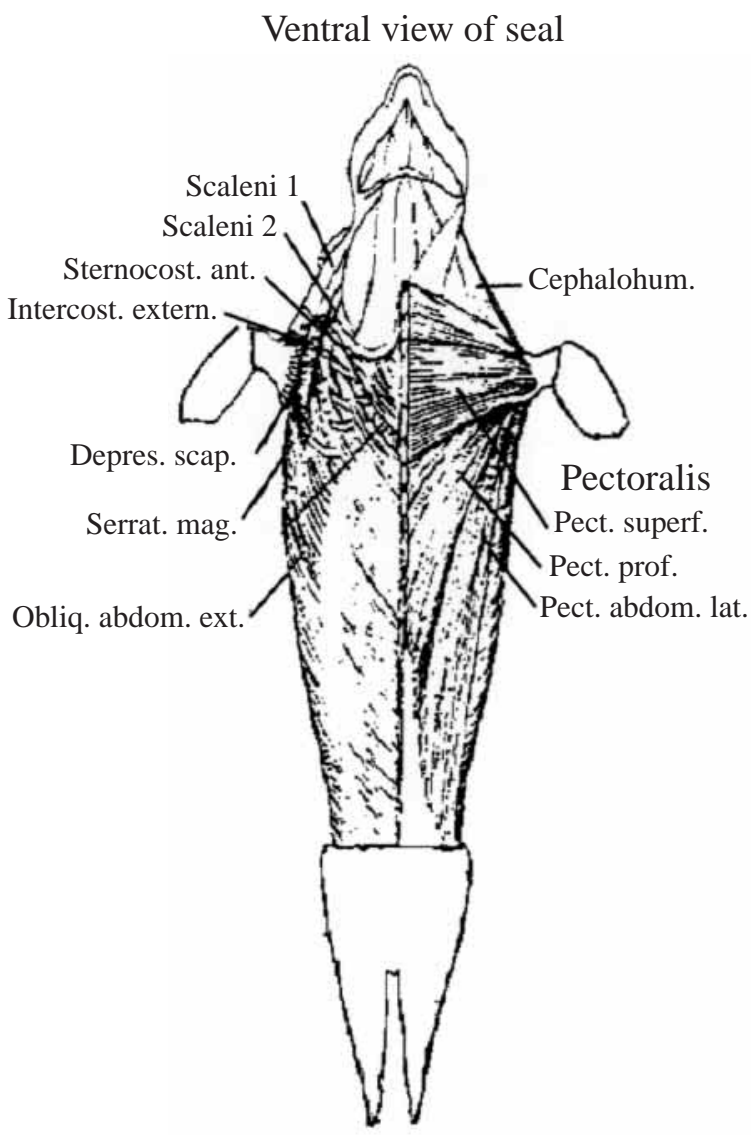

\section{Materials and methods}

Muscle preparation

Muscle samples were collected from 13 adult Weddell seals (Leptonychotes weddelli Lesson 1846, mean mass $=404 \pm 13.5 \mathrm{~kg}$ ) from McMurdo Sound, Antarctica. The seals were anesthetized by intramuscular injection of ketamine hydrochloride ( $2 \mathrm{mg} \mathrm{kg}^{-1}$; Davis et al., 1999). Three muscle samples of approximately $50 \mathrm{mg}$ each were collected with a 6mm biopsy cannula (Depuy, Warsaw, IN, USA) from both swimming (M. longissimus dorsi and hindlimb complex) and non-swimming (M. pectoralis) muscles (Fig. 1; Kanatous et al., 1999). Control samples were collected from the hindlimb muscles of dogs (Canis familiaris, $N=6$ ) anesthetized with pentobarbital sodium $\left(30 \mathrm{mg} \mathrm{kg}^{-1}\right)$. Immediately after collection, muscle samples were either: (1) placed into $2 \%$ glutaraldehyde fixative for electron and light microscopy, (2) frozen in liquid nitrogen for enzymatic and myoglobin assays or (3) mounted on cork and frozen in liquid-nitrogen-cooled isopentane for fiber-type histochemical analysis.

\section{Electron microscopy and morphometry}

Each glutaraldehyde-fixed muscle sample was cut into thin longitudinal strips, stored in glutaraldehyde fixative and processed for light and electron microscopy (EM), as previously described by Mathieu-Costello et al. (1992). Four

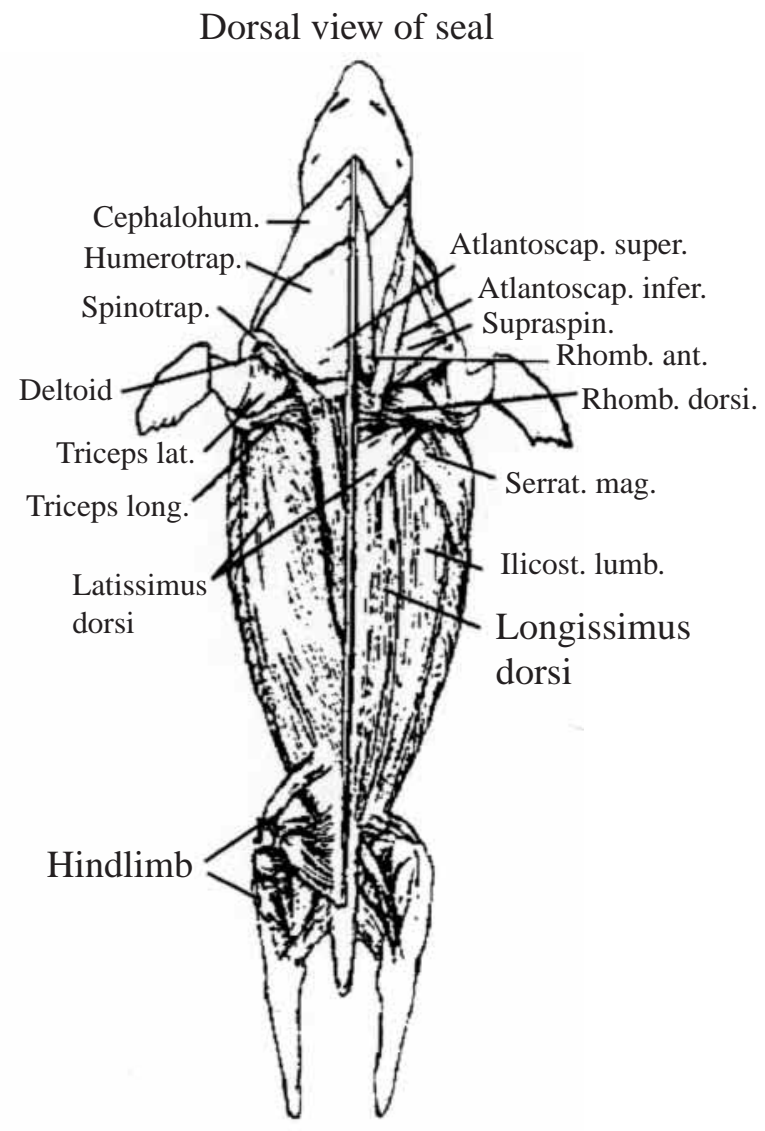

Fig. 1. Skeletal muscle anatomy of Weddell seals. Biopsy samples were collected from the swimming muscles (M. longissimus dorsi and hindlimb complex) and the non-swimming muscle (M. pectoralis). (Originally redrawn by M. Cooley, reproduced from Kanatous et al., 1999.) 
transverse sections $(1 \mu \mathrm{m})$ were collected from tissue blocks using an LKB Ultrotome III (Peapack, NJ, USA) and stained with $0.1 \%$ aqueous toluidine blue solution for light microscopy. Ultrathin sections $(50-70 \mathrm{~nm})$ were cut transversely to the muscle fiber axis in each block. They were contrasted with uranyl acetate and bismuth subnitrate, and electron micrographs for morphometry were taken on 70-mm film using a Zeiss 10 transmission electron microscope (Zeiss North America, Thornwood, NY, USA).

Capillary density $\left[Q_{\mathrm{A}}(0)\right.$; the number of capillaries per fiber cross-sectional area] was obtained by morphometric analysis on transverse sections using a 100-point eyepiece square-grid test system on $1 \mu \mathrm{m}$ thick transverse sections examined at a magnification of $400 \times$ with a light microscope. Capillary diameter $[\bar{d}(\mathrm{c})]$, average number of capillaries around a fiber $\left(N_{\mathrm{CAF}}\right)$ and mean fiber cross-sectional area $[\bar{a}(\mathrm{f})]$ were measured using an image analyzer (Videometric 150, American Innovision, San Diego, CA, USA) on the same transverse sections. $\bar{d}(\mathrm{c})$ was determined as the shorter axis of close-to-circular profiles only $(<20 \%$ difference between the shorter and longer diameters), which assumed capillary crosssectional circularity. Capillary-to-fiber ratio $\left[N_{\mathrm{N}}(\mathrm{c}, \mathrm{f})\right]$ was calculated as the product of $Q_{\mathrm{A}}(0)$ and $\bar{a}(\mathrm{f})$ (Mathieu-Costello, 1993).

The volume density of mitochondria, myofibrils and lipid droplets per volume of muscle fiber was estimated by pointcounting on electron micrographs obtained by systematic sampling in ultrathin transverse sections from each of the four tissue blocks from each sample. Contact prints of the EM film were projected at a final magnification of $24000 \times$ on a $144-$ point square-grid test system of a microfilm reader (Documator DL 2, Jenoptic, Jena, Germany).

\section{Enzymatic activities and myoglobin concentration}

Frozen muscle samples were thawed, weighed and homogenized at $0^{\circ} \mathrm{C}$ in buffer containing $1 \mathrm{mmoll}^{-1}$ EDTA, $2 \mathrm{mmoll}^{-1} \mathrm{MgCl}_{2}$ and $50 \mathrm{mmoll}^{-1}$ imidazole, $\mathrm{pH}$ 7.6. The homogenates were spun for $4-5 \mathrm{~min}$ at $10000 \mathrm{~g}$, and the supernatant was isolated. The assay conditions were as follows. Lactate dehydrogenase (LDH; EC 1.1.1.27): $50 \mathrm{mmoll}^{-1}$ imidazole; $0.15 \mathrm{mmoll}^{-1} \mathrm{NADH}, \mathrm{pH} 7.0$ at $37^{\circ} \mathrm{C}$; and $1 \mathrm{mmol}^{-1}$ pyruvate; $\Delta A_{340}$, millimolar extinction coefficient $\varepsilon_{340}=6.22$. $\beta$-hydroxyacyl CoA dehydrogenase (HAD; EC 1.1.1.35): $\quad 50 \mathrm{mmoll}^{-1} \quad$ imidazole; $1 \mathrm{mmoll}^{-1} \quad$ EDTA; $0.1 \mathrm{mmoll}^{-1}$ acetoacetyl $\mathrm{CoA}$; and $0.15 \mathrm{mmoll}^{-1} \mathrm{NADH}$, $\mathrm{pH} 7.0$ at $37^{\circ} \mathrm{C} ; \Delta A_{340}, \varepsilon_{340}=6.22$. Citrate synthase (CS; EC 4.1.3.7): $50 \mathrm{mmol}^{-1}$ imidazole; $0.25 \mathrm{mmol}^{-1} 5,5^{\prime}$-dithiobis(2nitrobenzoic acid) (DTNB); $0.4 \mathrm{mmoll}^{-1}$ acetyl $\mathrm{CoA}$; and $0.5 \mathrm{mmol}^{-1}$ oxaloacetate, $\mathrm{pH} 7.5$ at $37^{\circ} \mathrm{C} ; \Delta A_{412}, \varepsilon_{412}=13.6$. Specific enzyme activities $\left(\mu \mathrm{mol} \mathrm{min}^{-1} \mathrm{~g}^{-1}\right.$ wet mass of muscle) were calculated from the rate of change of the assay absorbance at the maximal linear slope. The enzyme ratio CS:HAD was calculated to assess the relative contribution of fatty acid metabolism to total aerobic metabolism.

Myoglobin was assayed according to the method of Reynafarje (1963) with the following modifications. A portion
$(500 \mu \mathrm{l})$ of the homogenate was further diluted with $1 \mathrm{ml}$ of phosphate buffer $\left(0.04 \mathrm{moll}^{-1}, \mathrm{pH} 6.6\right)$. The resulting mixture was centrifuged for $50 \mathrm{~min}$ at $28000 \mathrm{~g}$ at $4^{\circ} \mathrm{C}$. The supernatant was bubbled with carbon monoxide for $3 \mathrm{~min}$. Spectrophotometric absorbance was measured at $538 \mathrm{~nm}$ and $568 \mathrm{~nm}$, and the concentration of myoglobin in $\mathrm{mg} \mathrm{g}^{-1}$ wet mass of muscle was calculated as: (Abs538-Abs568) $\times$ $5.865[(1.5 / 0.5) \times($ mass of sample $)]$.

\section{Fiber type}

Cross sections of each muscle sample were cut into serial sections $(7-9 \mu \mathrm{m})$ with a Shandon cryotome (Thermo Shandon, Pittsburgh, PA, USA) maintained at $-20^{\circ} \mathrm{C}$. Sections were placed onto glass slides; four serial sections per slide. Transverse orientation was verified using a standard light microscope. Sections were stained using a metachromatic ATPase staining protocol modified from Olgive and Feeback (1990). Briefly, the procedure was as follows: (1) ATPase preincubation for $8 \mathrm{~min}(\mathrm{pH}$ of 4.5$)$ at room temperature, (2) three $2 \mathrm{~min}$ Tris rinses (pH7.8), (3) incubation with ATP for $25 \mathrm{~min}$ ( $\mathrm{pH} 9.4$ ), (4) three calcium chloride rinses, (5) counterstaining in $0.1 \%$ toluidine blue for $1 \mathrm{~min}$, (6) dehydration in ethanol and (7) clearing in xylene. The proportion of slow oxidative (Type I), fast-twitch oxidative (Type IIA) and fast-twitch glycolytic (Type IIB) fibers was determined by standard point counting procedure and is presented as percentages relative to the total number of fibers.

In addition to using dog hindlimb muscle controls, we further verified the validity of the metachromatic stain results in seal muscle by comparison with immunohistochemical staining for specific slow and fast myosin heavy chain isoforms after the reactivity of the antibodies with seal proteins had been verified by western analysis. Slides were fixed in ice-cold alcohol-formalin-acetic acid fixative in a Coplin jar and washed with phosphate-buffered saline (PBS). A proteinaceous blocking agent (Powerblock Reagent, Innogenex, San Ramon, CA, USA) was applied to each section to minimize non-specific antibody binding. A series of monoclonal antibodies specific to myosin heavy chain isoforms Type I, Type IIa and Type IIb were applied to one of the four sections on each of the slides and incubated overnight in a humidity chamber at $-4^{\circ} \mathrm{C}$. Serial amplification of the primary antibody was accomplished using an incubation of biotinylated secondary antibody for $20 \mathrm{~min}$, followed by a series of PBS washes followed by a 20 min incubation with alkaline-phosphatase streptavidin conjugate (Carson, 1990). After rinsing in PBS, Fast Red substrate was applied, and color development was stopped by washing in water. The slides were counterstained with Mayer's hematoxylin, washed in water, and cover slipped with Dako glycergel (Carpinteria, CA, USA). A sample of approximately 200-400 artifact-free and well-stained fibers was counted from each section using a camera-mounted microscope attached to a PC loaded with BIOQUANT software (Bioquant, Nashville, TN, USA) to determine the percentage of Type I, Type IIa, and Type IIb fibers in each sample. 


\section{Statistical analysis}

Statistical analysis was performed by analysis of variance (ANOVA) with Tukey post-hoc tests $(P \leq 0.05$, Sigmastat 2.0). Results are presented as means \pm S.E.M.

\section{Results \\ Skeletal muscle morphology}

The general morphology of the skeletal muscle fibers was similar between the swimming (M. longissimus dorsi and hindlimb complex) and non-swimming (M. pectoralis) muscles of Weddell seals and typical for mammals (Fig. 2). Electron micrographs of transverse sections showed irregularly shaped myofibrils, separated by sarcoplasmic reticulum, and mitochondria. The mean volume density of myofibrils per volume of muscle fiber was not significantly different among the swimming $(81.9 \pm 1.5 \%$ to $87.4 \pm 1.4 \%$ for the $\mathrm{M}$. longissimus dorsi and hindlimb, respectively) and nonswimming $(87.3 \pm 0.9 \%$ for the $\mathrm{M}$. pectoralis) muscles of the Weddell seal or between the muscles of the Weddell seal $(81.9 \pm 1.5 \%$ to $87.4 \pm 1.4 \%)$ and the $\operatorname{dog}(87.2 \pm 0.7 \%)$ (Table 1$)$.

There were no significant differences in the volume densities of mitochondria (total volume density of mitochondria, $2.78 \pm 0.21 \%$ to $3.13 \pm 0.28 \%$; volume density of subsarcolemmal mitochondria, $0.09 \pm 0.04 \%$ to $0.16 \pm 0.05 \%$; volume density of interfibrillar mitochondria, $2.62 \pm 0.18 \%$ to $3.01 \pm 0.28 \%)$ or lipid droplets $(0.06 \pm 0.05 \%$ to $0.16 \pm 0.07 \%)$ among the muscles of the Weddell seal (Table 1). However, the total volume density of mitochondria $(5.9 \pm 0.5 \%)$, the volume density of subsarcolemmal mitochondria $(0.6 \pm 0.1 \%)$ and the volume density of interfibrillar mitochondria $(5.3 \pm 0.4 \%)$ in the hindlimb of the dog were significantly greater than in the muscles of the Weddell seal (Table 1). By contrast, there was no significant difference in the volume density of intracellular lipid droplets between the muscles of Weddell seals $(0.06 \pm 0.05 \%$ to $0.16 \pm 0.07 \%)$ and dog hindlimb muscle $(0.1 \pm 0.0 \%)$ (Table 1$)$.

The M. longissimus dorsi, the principal swimming muscle of the seal, had a significantly greater fiber cross-sectional area and lower capillary density than the M. pectoralis and hindlimb

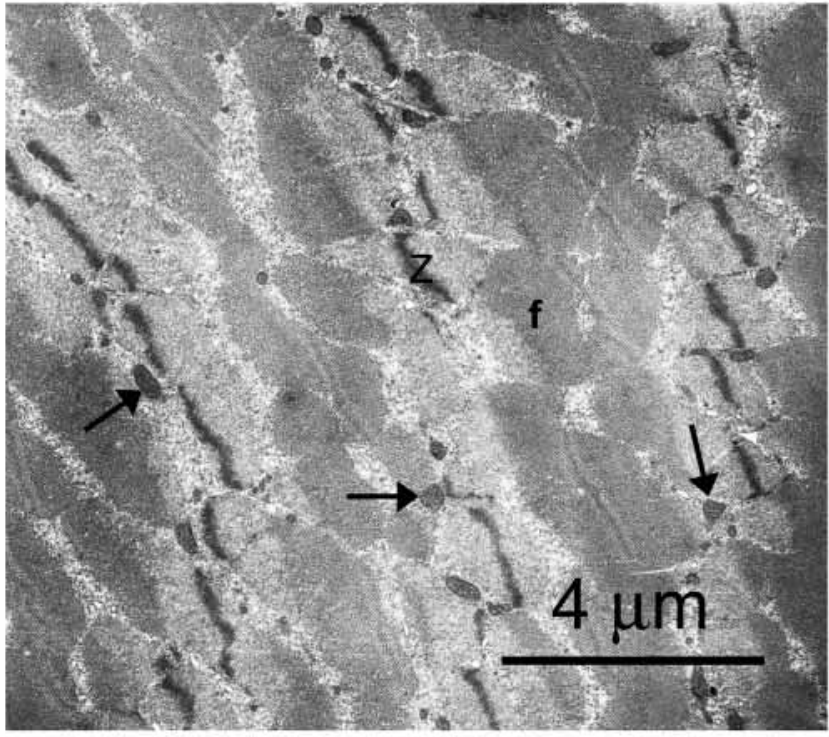

Fig. 2. Representative electron micrograph of the muscles from the Weddell seal. A transverse section through the hindlimb muscle, showing myofibrils (f), Z line (Z) and mitochondria (arrows).

muscle complex (Table 2). By contrast, there were no significant differences among the muscle groups of the Weddell seal in indices of capillary supply that were independent of fiber area (i.e. capillary-to-fiber ratio and average number of capillaries around a fiber) (Table 2). While the fiber cross-sectional areas of the Weddell seal muscles were significantly greater than those in the dog hindlimb, the indices of capillarity were significantly less (Table 2).

\section{Enzyme activities}

As with the volume density of mitochondria, there were no significant differences in the activities of the aerobic enzymes citrate synthase (CS) and $\beta$-hydroxyacyl CoA dehydrogenase (HAD) between swimming and non-swimming muscles of the seals. However, CS activity and HAD activity in seal muscle were significantly lower $(47-50 \%)$ and significantly greater $(2.3-4.1 \times)$, respectively, than in dog hindlimb muscle

Table 1. Morphometric estimates for fiber ultrastructure in Weddell seals

\begin{tabular}{|c|c|c|c|c|c|c|}
\hline Animal & Muscle & $\begin{array}{c}V_{\mathrm{v}}(\mathrm{mt}, \mathrm{f}) \\
\%\end{array}$ & $\begin{array}{c}V_{\mathrm{v}}(\mathrm{ms}, \mathrm{f}) \\
\%\end{array}$ & $\begin{array}{c}V_{\mathrm{v}}(\mathrm{mi}, \mathrm{f}) \\
\%\end{array}$ & $\begin{array}{c}V_{\mathrm{v}}(\mathrm{li}, \mathrm{f}) \\
\%\end{array}$ & $\begin{array}{c}V_{\mathrm{v}}(\mathrm{my}, \mathrm{f}) \\
\%\end{array}$ \\
\hline $\begin{array}{l}\text { Weddell seal } \\
\qquad(N=13)\end{array}$ & $\begin{array}{l}\text { Longissimus } \\
\quad \text { dorsi } \\
\text { Pectoralis } \\
\text { Hindlimb }\end{array}$ & $\begin{array}{l}3.13 \pm 0.28 \\
2.78 \pm 0.21 \\
2.89 \pm 0.17\end{array}$ & $\begin{array}{l}0.09 \pm 0.04 \\
0.14 \pm 0.06 \\
0.16 \pm 0.05\end{array}$ & $\begin{array}{l}3.01 \pm 0.28 \\
2.62 \pm 0.18 \\
2.74 \pm 0.16\end{array}$ & $\begin{array}{l}0.16 \pm 0.07 \\
0.06 \pm 0.05 \\
0.13 \pm 0.04\end{array}$ & $\begin{array}{c}81.90 \pm 1.50 \\
87.3 \pm 0.9 \\
87.4 \pm 1.4\end{array}$ \\
\hline $\begin{array}{l}\text { Dog } \\
\qquad(N=6)\end{array}$ & Hindlimb & $5.9 \pm 0.5^{*}$ & $0.6 \pm 0.1 *$ & $5.3 \pm 0.4 *$ & $0.1 \pm 0.0$ & $87.2 \pm 0.7$ \\
\hline
\end{tabular}

Values are means \pm S.E.M. *Significantly different from Weddell seal $(P<0.05)$. $V_{\mathrm{v}}(\mathrm{mt}, \mathrm{f})$, total volume density of mitochondria; $V_{\mathrm{v}}(\mathrm{ms}, \mathrm{f})$, volume density of subsarcolemmal mitochondria; $V_{\mathrm{v}}(\mathrm{mi}, \mathrm{f})$, volume density of interfibrillar mitochondria; $V_{\mathrm{v}}(\mathrm{li}, \mathrm{f})$, volume density of intracellular lipid droplets; and $V_{\mathrm{v}}(\mathrm{my}, \mathrm{f})$, volume density of myofibrils; all quantities expressed per fiber volume. Dog data was previously reported in Kanatous et al., 2001. 
Table 2. Morphometric estimates of fiber cross-sectional area and capillarity in Weddell seals

\begin{tabular}{|c|c|c|c|c|c|}
\hline Animal & Muscle & $\begin{array}{c}\bar{a}(\mathrm{f}) \\
\left(\mu \mathrm{m}^{2}\right)\end{array}$ & $\begin{array}{c}Q_{\mathrm{A}}(0) \\
\left(\mathrm{mm}^{-2}\right)\end{array}$ & $N_{\mathrm{N}}(\mathrm{c}, \mathrm{f})$ & $N_{\mathrm{CAF}}$ \\
\hline \multirow[t]{2}{*}{ Weddell seal $(N=13)$} & Longissimus dorsi & $6992 \pm 539^{\dagger}$ & $207 \pm 8^{\dagger}$ & $1.45 \pm 0.12$ & $3.25 \pm 0.24$ \\
\hline & Hindlimb & $5386 \pm 584$ & $279 \pm 18$ & $1.46 \pm 0.12$ & $3.46 \pm 0.24$ \\
\hline $\operatorname{Dog}(N=6)$ & Hindlimb & $1530 \pm 125^{*}$ & $1617 \pm 104 *$ & $2.42 \pm 0.10^{*}$ & $5.96 \pm 0.17 *$ \\
\hline
\end{tabular}

Values are means \pm S.E.M. ${ }^{\dagger}$ Significantly different from pectoralis and hindlimb muscles in Weddell seal. *Significantly different from Weddell seal $(P<0.05) . \bar{a}(\mathrm{f})$, mean fiber cross-sectional area; $Q_{\mathrm{A}}(0)$, number of capillaries per fiber cross-sectional area; $N_{\mathrm{N}}(\mathrm{c}$,f), capillary-tofiber ratio; $N_{\mathrm{CAF}}$, average number of capillaries around a fiber. Dog data was previously reported in Kanatous et al., 2001.

(Table 3). In the Weddell seal, lactate dehydrogenase (LDH) activity, an index of anaerobic capacity, was significantly lower $(45 \%)$ in the hindlimb muscle complex than in the M. pectoralis and was similar to that measured in the hindlimb of the dog. The CS:HAD ratio, an index of the contribution of fatty acid metabolism to total aerobic metabolism, ranged from 0.2 to 0.4 in the muscles of Weddell seals, which indicated a complete reliance on fatty acids as the main fuel source for aerobic metabolism. In other words, all of the carbon flux through the Krebs cycle could be accounted for by carbon generated from fatty acid catabolism.

\section{Myoglobin}

The concentrations of myoglobin were not significantly different among swimming muscles and non-swimming muscles of Weddell seals; however, they were significantly greater (8-13-fold) than in the hindlimb of the dog.

\section{Fiber type}

There were significantly greater percentages of slow oxidative (Type I) fibers in the swimming muscles $(67 \pm 4.7 \%$ and $68.7 \pm 4.7 \%$ for the M. longissimus dorsi and hindlimb, respectively) as compared with non-swimming muscle (41.0 $\pm 5.4 \%$ for the M. pectoralis) (Figs 3, 4). Conversely, there was a significantly greater percentage of fast-twitch oxidative (Type IIA) fibers in the non-swimming $(58.8 \pm 5.4 \%$ for the M. pectoralis) as compared with the swimming muscles (33.6 $\pm 5.6 \%$ and $31.3 \pm 4.7 \%$ for the M. longissimus dorsi and hindlimb, respectively). There was a complete absence of fast- twitch glycolytic (Type IIB) fibers in the seal muscles. These results were consistent using both the histochemical technique for myosin ATPase activity and specific immunohistochemical staining for slow and fast myosin heavy chain isoforms.

\section{Discussion}

Despite routine exposure to hypoxia and ischemia associated with breath-hold diving, the muscles of Weddell seals are adapted for low levels of aerobic, lipid-based metabolism. Conversely, shorter-duration divers, such as harbor seals and Steller sea lions, that experience both hypoxia and relatively high rates of oxygen consumption during diving exhibit enhanced aerobic capacities in their skeletal muscles to maintain an aerobic lipid-based metabolism. This is facilitated by a suite of skeletal muscle adaptations that include increased volume densities of mitochondria in their swimming muscles, with an adaptive increase in interfibrillar mitochondria to reduce diffusion distance within the muscles, enhanced aerobic capacities similar to those of athletic terrestrial mammals of comparable size, elevated volume density of intrafiber lipid droplets and elevated concentrations of myoglobin in their skeletal muscles to offset their lower capillary supply (Kanatous et al., 1999, 2001). Data from the present study demonstrate that long-duration divers such as the Weddell seals display some but not all of the same adaptations as the short-duration divers. Fig. 5 displays the plot of muscle mitochondrial volume density against body mass from terrestrial and diving mammals. The linear relationship

Table 3. Enzyme activities and myoglobin concentration in skeletal muscle of Weddell seals

\begin{tabular}{lcccc}
\hline & & Weddell seal & Dog \\
& Longissimus dorsi & pectoralis & Hindlimb & hindlimb \\
\hline Citrate synthase (CS) & $12.0 \pm 1.3$ & $12.4 \pm 0.8$ & $11.6 \pm 1.9$ & $24.8 \pm 3.3^{\dagger}$ \\
B-hydroxyacyl Co A dehydrogenase (HAD) & $48.5 \pm 9.0$ & $38.9 \pm 7.5$ & $67.6 \pm 11.0$ & $16.6 \pm 2.8^{\dagger}$ \\
Lactate dehydrogenase (LDH) & $563 \pm 89$ & $739 \pm 66$ & $331 \pm 75^{*}$ & $396 \pm 38^{\dagger}$ \\
CS:HAD & 0.3 & 0.3 & 0.2 & 1.5 \\
Myoglobin & $45.9 \pm 3.3$ & $31.5 \pm 4.6$ & $42.5 \pm 5.7$ & $3.5^{\dagger}$
\end{tabular}

Values are means \pm S.E.M. *Significantly different from pectoralis; ${ }^{\dagger}$ significantly different from the Weddell seal; ${ }^{\dagger}$ significantly different from Longissimus dorsi and pectoralis. Enzyme activities are in $\mu \mathrm{mol} \mathrm{min} \mathrm{g}^{-1}$ wet mass of muscle. 

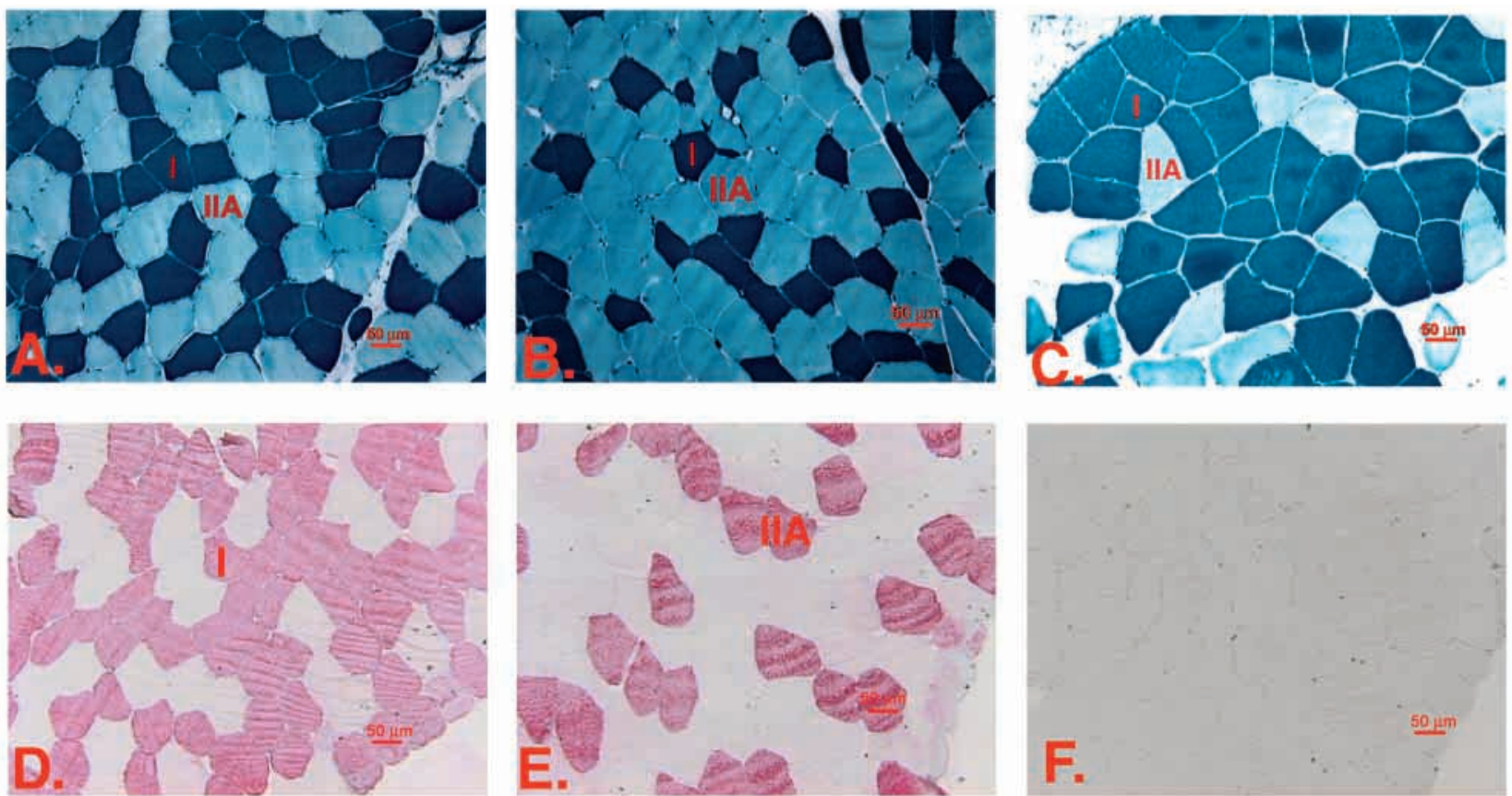

Fig. 3. Fiber-type composition in the muscles of Weddell seals. (A-C) Representative sections from metachromatic ATPase-stained muscle samples from (A) M. longissimus dorsi (swimming muscle), (B) M. pectoralis (non-swimming muscle) and (C) hindlimb (swimming muscle). I, Type I (slow oxidative) fibers; IIA, Type IIA (fast-twitch oxidative) fibers; Type IIB (fast-twitch glycolytic) fibers are completely absent. (D-F) Representative sections of specific immunohistochemical staining of the M. longissimus dorsi for the different fiber types. Darkly stained fibers are positive for: (D) Type I myosin (slow oxidative) and (E) Type IIA myosin (fast-twitch oxidative). (F) Type IIB myosin (fasttwitch glycolytic) are completely absent.

$\left(y=6.75-1.34[\log x], r^{2}=0.70\right)$ was generated from the volume densities of the vastus medialis, a primary locomotory muscle, from various terrestrial mammals ranging in size from the dwarf mongoose (Helogale pervula) to the steer (Bos taurus) (Kanatous et al., 1999; and terrestrial data from Hoppeler et

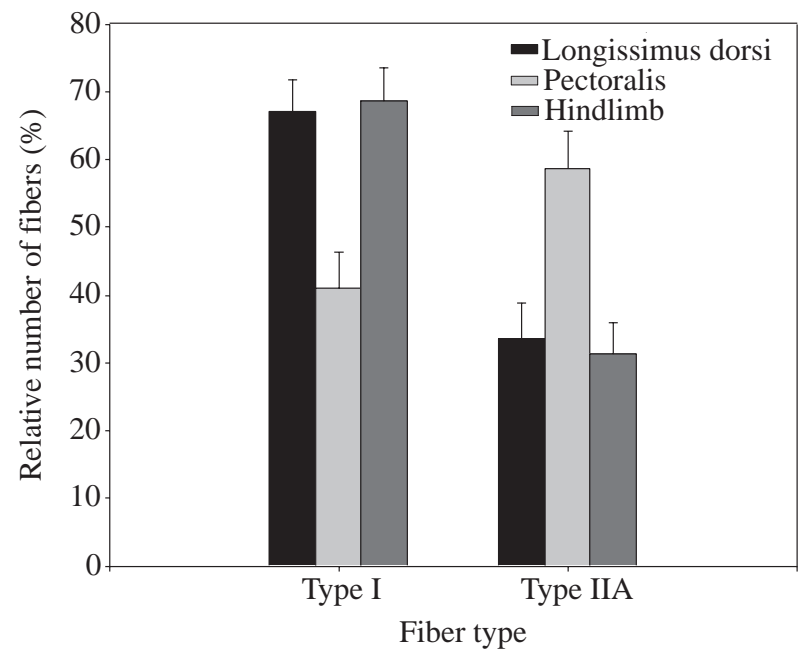

Fig. 4. Histogram showing fiber-type composition (\% of total fiber number) in the swimming (M. longissimus dorsi and hindlimb) and non-swimming (M. pectoralis) muscles of Weddell seals. Note the lack of fast-twitch glycolytic (Type IIB) fibers in all the muscles measured. al., 1987). While the skeletal muscles of shorter-duration divers had enhanced aerobic capacities similar to those found in terrestrial animal athletes, such as the dog and pony (Equus caballus), the volume density of mitochondria in the swimming muscle (M. longissimus dorsi) of Weddell seals was similar to that predicted for a sedentary terrestrial mammal of comparable size. These results are similar to those found in another deep, long-duration diver; the emperor penguin (Aptenodytes forsteri). Ponganis et al. (1997) found volume densities of mitochondria in the swimming muscles of emperor penguins similar to those of the locomotory muscles of sedentary terrestrial mammals of comparable size but significantly less than athletic terrestrial mammals of comparable size. These results for the penguin are consistent with our measurements of low levels (i.e. 1-2x resting metabolic rate) of energy metabolism in Weddell seals during aerobic dives (Williams et al., 2000).

The mitochondrial volume densities and activities of aerobic enzymes in the swimming muscles of Weddell seals were not significantly different from those of the nonswimming muscles (Table 1). While the total volume density of mitochondria did not differ from that of sedentary terrestrial mammals, the distribution of the mitochondria throughout the muscles did. As shown previously for shallow divers, the majority of mitochondria were interfibrillar, with less than 5\% of total mitochondrial volume density being subsarcolemmal. By contrast, subsarcolemmal mitochondria usually account 


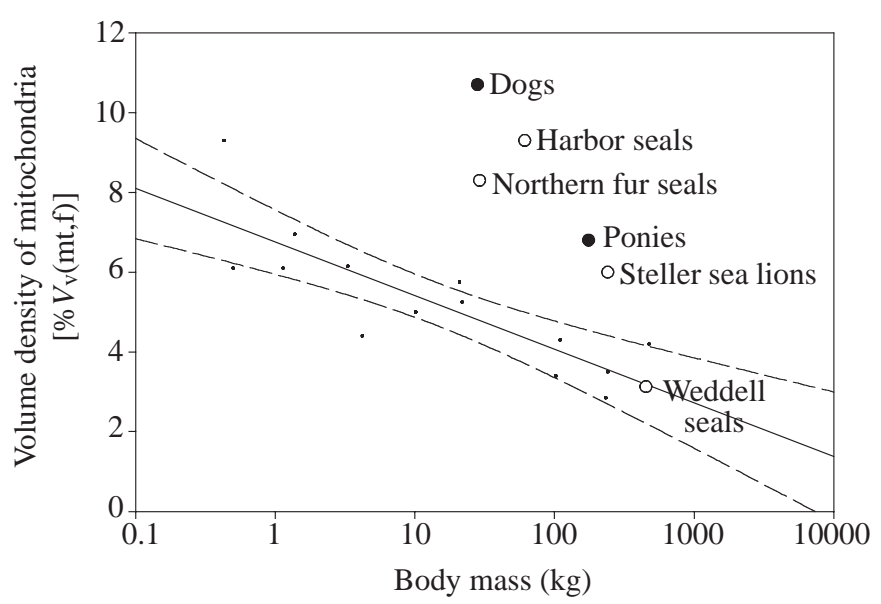

Fig. 5. Plot of muscle mitochondrial volume density against body mass in athletic (filled circles) and sedentary terrestrial mammals (small dots) and pinnipeds (open symbols). The linear relationship $\left(y=6.75-1.34[\log x], \quad r^{2}=0.70\right)$ was generated from the volume densities of the vastus medialis, a primary locomotory muscle, from various terrestrial mammals ranging in size from the dwarf mongoose to the steer (Kanatous et al., 1999; and terrestrial data from Hoppeler et al., 1987). By contrast to the shorter-duration divers, the volume density of mitochondria in the M. longissimus dorsi of Weddell seals was similar to that predicted for a sedentary terrestrial mammal of comparable size.

for $\approx 10 \%$ of the total mitochondrial volume in sedentary terrestrial mammals and in excess of $30 \%$ in athletic species (Kayar et al., 1989). Thus, while Weddell seal total volume density of mitochondria did not differ from that of terrestrial mammals, there was a difference in the distribution of mitochondria throughout the muscle. Another similarity between the short- and long-duration divers was a reliance on fatty acids as their principal fuel source for aerobic metabolism, as evidenced by CS:HAD ratios, which were substantially less than 1 (Table 3).

Although the muscles of Weddell seals appear to be geared for lipid-based, aerobic metabolism, they have a substantially reduced capillary supply compared with that of terrestrial mammals. Kanatous et al. (2001) found that the capillary density in the swimming muscles of harbor seals was approximately $50 \%$ less than in hindlimb muscles of dogs with similar volume densities of mitochondria. The same pattern is found in Weddell seals [body mass $=404 \pm 13.5 \mathrm{~kg}$; $Q_{\mathrm{A}}(0)=259.8 \pm 26.7 \mathrm{~mm}^{2}$ ] compared with terrestrial mammals of comparable size, such as the horse Equus equus [mean body mass $=447 \pm 36 \mathrm{~kg} ; Q_{\mathrm{A}}(0)=926 \pm 36 \mathrm{~mm}^{2}$ ] or the steer [mean body mass $=474 \pm 12 \mathrm{~kg} ; Q_{\mathrm{A}}(0)=727 \pm 6 \mathrm{~mm}^{2}$ ] (Kayar et al., 1992). This paradoxical result (a high reliance on aerobic metabolism with diminished capillary supply) is explained by the high concentration of myoglobin in Weddell seal muscles (Table 3). The elevated myoglobin concentrations appear to offset the diminished capillary supply by increasing both the endogenous oxygen storage capacity and the apparent intracellular diffusing capacity of oxygen in the muscle, allowing aerobic metabolism to be maintained under the conditions of low oxygen partial pressures and ischemia resulting from the dive response.

Interestingly, the fiber-type distribution of the Weddell seals, as determined by a metachromatic ATPase-based stain and immunohistochemical techniques, revealed a complete absence of fast-twitch glycolytic fibers (type IIB) in both swimming and non-swimming muscles (Figs 3, 4). To our knowledge, this is the first reported muscle-fiber-type analysis for Weddell seals. Previous studies on short-duration divers found a generally mixed fiber type that included all three major fiber classifications in the muscles (Reed et al., 1994; Kanatous et al., 1999). The fiber-type profile of the Weddell seals was consistent with our results for LDH activity, an indicator of anaerobic capacity. The hindlimb was composed of $\approx 69 \%$ slow oxidative fibers and had the lowest activity of $\mathrm{LDH}$, followed by the M. longissimus dorsi and then the M. pectoralis, which had only $41 \%$ slow oxidative fibers and the highest LDH activity (Fig. 4, Table 3). While the lack of Type IIB fibers in muscles of Weddell seals was unexpected, it adds support to the hypothesis that they preferentially maintain low levels of aerobic metabolism during the majority of dives.

With advances in minimizing the size of electronics, an animal-borne video system and data recorder was developed and deployed on different species of marine mammals, including Weddell seals. This system permitted direct observation of diving behavior and foraging strategy (Davis et al., 1999; Williams et al., 2000), allowing assessment of the locomotor strategies used by marine mammals throughout their dives. Simultaneous measurements of metabolic rate and flipper-stroke frequency provided new information on energyconserving modes of locomotion in diving Weddell seals. In previous studies, swim speed was considered to be an important indicator of energetic cost. However, we observed different energetic costs for gliding (no stroking) during descent, intermittent stroking during horizontal travel and continuous stroking during ascent, even though these activities occur at similar speeds $\left(\approx 2.0 \mathrm{~m} \mathrm{~s}^{-1}\right)$. Prolonged gliding (1-9 min in duration), which appears to be associated with changes in buoyancy during descent, resulted in significant energy savings. In addition to Weddell seals, flipper-stroke frequency and swim speed have been measured in a northern elephant seal (Mirounga angustirostris) and bottlenose dolphins (Tursiops truncatus) (Williams et al., 2000). These two species also exhibited periods of prolonged gliding during descent. Although swim speed has been used as an indicator of effort, our research has shown stroke frequency to be a better indicator of energy expenditure. It showed that marine mammals take advantage of a number of physical properties of the aquatic environment and use behavioral strategies that reduce the energetic costs of diving (e.g. up to $59.6 \%$ in Weddell seals) and that deep divers employ these techniques more often or to a greater extent than do shallow divers. These energetic savings allow marine mammals to increase their dive durations beyond their predicted ADL (based on calculations of oxygen consumption and body oxygen stores) and achieve 
remarkable depths despite limited oxygen availability during diving (Williams et al., 2000).

In summary, the skeletal muscles of Weddell seals do not have enhanced aerobic capacities compared with those of terrestrial mammals but are adapted to maintain low levels of an aerobic lipid-based metabolism, especially under the hypoxic conditions associated with diving. This is in contrast to shorter-duration, active divers that exhibit aerobic capacities in their skeletal muscles that are similar to those of athletic terrestrial mammals. In other words, the combination of diving hypoxia and moderate levels of exercise appears to enhance aerobic capacity in their muscles to a degree seen in terrestrial animal athletes such as the dog and pony with much greater exercise capacity. The lower aerobic capacity of Weddell seal muscle reflects their energyconserving modes of locomotion (via extended periods of gliding during descent) that enable longer, deeper dives. Based on these results, the skeletal muscles of marine mammals appear to adapt to the hypoxic conditions of diving in different manners.

This work was supported by the National Heart, Lung and Blood Institutes grant PO1 HL 17731 and by the NSF Division of Polar Programs (grant OPP 14857). We wish to thank Ann Bull, Peter Agey, Larnele Hazelwood, Li Wu and Susanne Kohin for their assistance in various aspects of this study.

\section{References}

Burns, J. M. (1999). The development of diving behavior in juvenile Weddell seals: pushing limits in order to survive. Can. J. Zool. 77, 737-747.

Carson, F. L. (1990). Histotechnology: A Self-Instructional Text. Chicago, IL, USA: ASCP Press.

Castellini, M. A., Davis, R. W., Davis, M. P. and Horning, M. (1984). Antarctic marine life under the McMurdo ice shelf at White Island: A link between nutrient influx and seal population. Polar Biol. 2, 229-231.

Castellini, M. A., Davis, R. W. and Kooyman, G. L. (1992). Annual Cycles of Diving Behavior and Ecology of the Weddell Seal. Bulletin of the Scripps Institution of Oceanography, vol. 28. Berkeley, CA, USA: University of California Press.

Davis, R. W., Castellini, M. A., Williams, T. M. and Kooyman, G. L. (1991). Fuel homeostasis in the harbor seal during submerged swimming. J. Comp. Physiol. B 160, 627-635.

Davis, R. W. and Kanatous, S. B. (1999). Convective oxygen transport and tissue oxygen consumption in Weddell seals during aerobic dives. J. Exp. Biol. 202, 1091-1113.

Davis, R. W., Fuiman, L. A., Williams, T. M., Collier, S. O., Hagey, W. P., Kanatous, S. B., Kohin, S. and Horning, M. (1999). Hunting behavior of a marine mammal beneath the Antarctic fast ice. Science 283, 993-996.

Elsner, R., Oyasaeter, S., Almaas, R. and Didrik Saugstad, O. (1998).
Diving seals, ischemia-reperfusion and oxygen radicals. Comp. Biochem Physiol. A 119, 975-980.

Guyton, G. P., Stanek, K. S., Schneider, R. C., Hochachka, P. W., Hurford, W. E., Zapol, D. G., Liggins, G. C. and Zapol, W. M. (1995). Myoglobin saturation in free-diving Weddell seals. J. Appl. Physiol. 79, 1148-1155.

Hochachka, P. W. (1986). Balancing conflicting metabolic demands of exercise and diving. Federation Proc. 45, 2948-2952.

Hochachka, P. W. (1992). Metabolic biochemistry of a mesopelagic mammal. Experentia 48, 570-574.

Hoppeler, H., Kayar, S. R., Claassen, H., Uhlmann, E. and Karas, R. H. (1987). Adaptive variation in the mammalian respiratory system in relation to energetic demand: III. Skeletal muscles set the demand for oxygen. Resp. Phys. 69, 27-46.

Kanatous, S. B., DiMichele, L. V., Cowan, D. F. and Davis, R. W. (1999). High aerobic capacities in the skeletal muscles of pinnipeds: adaptations to diving hypoxia. J. Appl. Physiol. 86, 1247-1256.

Kanatous, S. B., Elsner, R. and Mathieu-Costello, O. (2001). Muscle capillary supply in harbor seals. J. Appl. Physiol. 90, 1919-1926.

Kayar, S. R., Hoppeler, H., Lindstedt, S. L., Classen, H., Jones, J. H., Essen-Gustavsson, B. and Taylor, C. R. (1989). Total mitochondrial volume in relation to aerobic capacity of horses and steers. Pflügers Arch. 413, 343-347.

Kayar, S. R., Hoppeler, H., Lindstedt, S. L., Classen, H., Jones, J. H., Essen-Gustavsson, B. and Taylor, C. R. (1992). Estimating transit time for capillary blood in selected muscles of exercising animals. Pflügers Arch. 421, 578-582.

Kooyman, G. L. (1981). Weddell Seal, Consummate Diver. New York: Cambridge University Press.

Kooyman, G. L. and Ponganis, P. J. (1998). The physiological basis of diving to depth: birds and mammals. Annu. Rev. Physiol. 60, 19-32.

Kooyman, G. L., Ponganis, P. J. and Howard, R. S. (1999). Diving Animals. In The Lung at Depth (ed. C. E. G. Lundgren and J. N. Miller), pp. 587620. New York: Marcel Dekker, Inc.

Mathieu-Costello, O., Szewczak, J. M., Logemann, R. B. and Agey, P. J. (1992). Geometry of blood-tissue exchange in bat flight muscle compared with bat hindlimb and rat soleus muscle. Am. J. Physiol. 262, R955-R965.

Mathieu-Costello, O. (1993). Comparative aspects of muscle capillary supply. Annu. Rev. Physiol. 55, 503-525.

Olgive, R. W. and Feeback, D. L. (1990). A metachromatic dye-ATPase method for the simultaneous identification of skeletal muscle fiber types I, IIA, IIB and IIC. Stain Technol. 65, 231-241.

Ponganis, P. J., Costello, M. L., Starke, L. N., Mathieu-Costello, O. and Koooyman, G. L. (1997). Structural and biochemical characteristics of Aptenodytes forsteri. Resp. Physiol. 109, 73-80.

Reed, J. Z., Butler, P. J. and Fedak, M. A. (1994). The metabolic characteristics of the locomotory muscles of grey seals (Halichoerus grypus), harbour seal (Phoca vitulina), and Antarctic fur seals (Arctocephalus gazella). J. Exp. Biol. 194, 33-46.

Reynafarje, B. (1963). Simplified method for the determination of myoglobin. J. Lab. Clin. Med. 61, 138-145.

Testa, J. W. (1994). Overwinter movements and diving behavior of female Weddell seals (Leptonychotes weddelli) in the SW Ross Sea, Antarctica. Can. J. Zool. 72, 1700-1710.

Wartzok, D., Elsner, R., Stone, H., Kelly, B. P. and Davis, R. W. (1992). Under-ice movements and the sensory basis of hole finding behavior in ringed and Weddell Seals. Can. J. Zool. 70, 1712-1722.

Williams, T. M., Davis, R. W., Fuiman, L. A., Francis, J., Le Boeuf, B. J., Horning, M., Calambokidis, J. and Croll, D. A. (2000). Sink or swim: strategies for cost-effective diving by marine mammals. Science 288, 133136. 